\title{
Development and Description of a Retail Outlet Simulation Model for Validating Information System Algorithms
}

\section{M.A. Dubova and A.N. Kulemin}

JSC ABC Solutions, Russia, Moscow, Letnikovskaya st., 10/4

\section{Abstract}

The article discusses the process of development an imitational model of the outlet. This model is created to validate calculation algorithms in the workforce management information system by evaluating the effectiveness of the some count of schedules, which are built with this system. If this schedules are more effective than the previous algorithm's schedules, so new algorithm is more effective than previous version. Model is needed because calculation algorithms can not be used for validation themselves. Validation with real client's outlet is so long and expensive, and is check only one or pair the calculation's algorithms results, so in fact it can't be used. Imitation model

Corresponding Author: M.A. Dubova

mariya.dubova.97@mail.ru

Received: 5 March 2020

Accepted: 18 March 2020

Published: 8 April 2020

Publishing services provided by Knowledge E

(c) M.A. Dubova and A.N.

Kulemin. This article is

distributed under the terms of the Creative Commons

Attribution License, which permits unrestricted use and redistribution provided that the original author and source are credited.

Selection and Peer-review under the responsibility of the SEC 2019 Conference Committee.

\section{G OPEN ACCESS} provide a chance to imitate works different types of outlets with many variants results of new version the calculation algorithms. AnyLogic was chosen because it is superior in functionality to the alternative despite the rather high cost of an official license. The text contains the results of the work performed, a brief description of the stages is given.

Keywords: simulation modeling, AnyLogic, validation, information systems, workforce management

\section{Introduction}

A model is a physical or abstract object endowed with the most important properties of a real object within the framework of this task. Modeling is the process of building a model in order to further study the properties and behavior of the studied object (real system).The purpose of simulation is to reproduce the behavior of the system under study using the basic relationships between its elements. [1]

Simulation is often used at the stage of analysis and refinement of a set of system requirements as a form of experimental testing. It allows you to understand how the system will behave even before its physical embodiment. Simulation makes it possible to obtain the information necessary for designing much faster and at a lower cost than in the case of building and testing system components. Modeling can help in the study of emergency and non-standard situations: by changing key indicators in the model, 
specialists can predict the behavior of the system. Simulation models are dynamic, that is, they describe behavior over time. [2]

Simulation can be successfully used to validate the mathematical algorithms of WFM class systems, the main task of whose is to increase the efficiency of human resource management by optimizing payroll and the level of service in retail.

The principle of operation of scheduling systems is usually as follows. Based on the historical data on traffic and the number of checks provided by the client, taking into account the specified parameters: the number of staff units, the store's working hours, the system calculates the traffic forecast and resource requirements. Then, taking into account the obtained values and preferences of the workers by the number of shifts at a certain time of the day, the optimal schedule option is determined. This obtained artifact can serve as an object of study within the framework of the process of validating information system algorithms.

\section{Process Description}

For the timely validation of the mathematical algorithms of the WFM system by comparing two variants of employee work schedules, it was decided to use simulation modeling.

To build a simulation model, you must take the following steps:

1. to analyze the structure of the simulated process.

2. to determine the input and output parameters of the model.

3. to decide on the choice of simulation tools.

4. to build a graph of the model using the constructor.

5. to set parameter values for graph nodes.

6. to determine the conditions for choosing a node if there are branches in the model.

7. to prepare a set of input data.

8. to compile the model.

The simulated process is the following sequence of actions.

The client enters the trading floor. If at this moment there are free employees, the buyer receives a consultation. We agree that the probability of making a purchase after talking with a consultant increases. If the client found what he was looking for, then he goes to the cashier; if the characteristics of the goods don't suit him, he leaves the store. If there are enough cashiers to provide timely service to all customers, the customer makes a purchase and leaves the store. If the queue is too long, it is likely 
that the potential buyer won't want to spend time waiting and may change his decision to purchase the goods.

In the absence of a consultant, the client reviews the goods on their own. The probability of finding the right product is reduced in this case. If the client has a desire to make a purchase, then he moves to the cash register, where he pays for the goods if there are enough cashiers.

Such initial data are required as the employee schedule (number of people in each group per unit of time), outlet parameters (hours of operation, number of employees, type of customer service), historical data on traffic (number of customers) and checks per unit time to simulate the customer service process. Based on the simulation results, an integral assessment of each of the work schedules loaded into the model will be obtained. This assessment consists of quantitative values of performance indicators and their weighting factors.

The Figure 1 shows a functional model of the target system in the form of a "black box". There are input and output streams on the diagram.

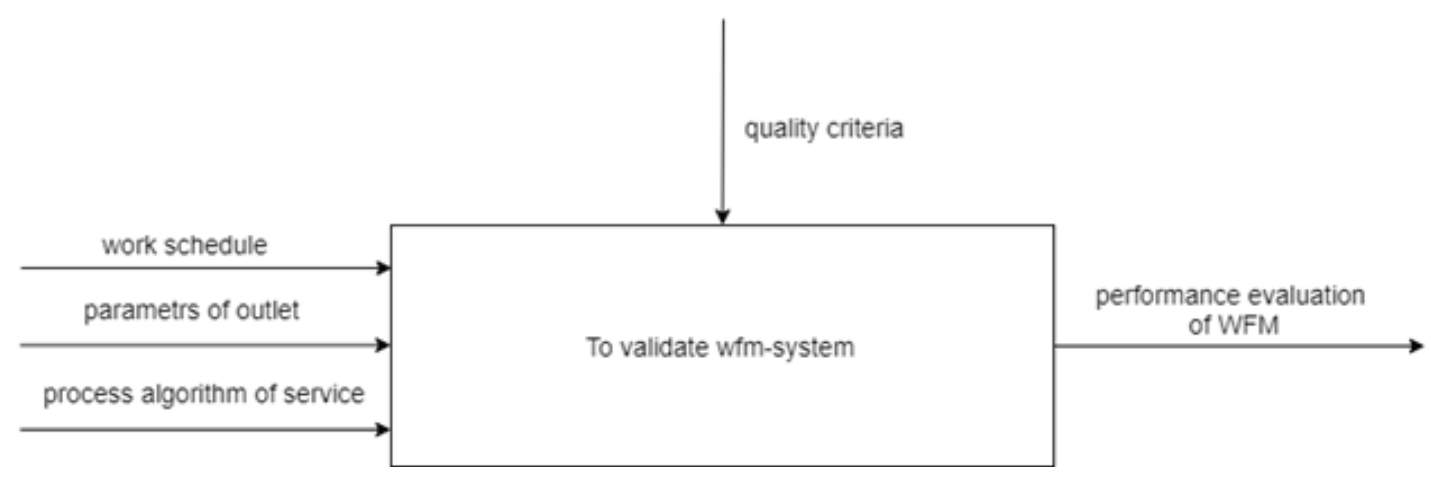

Figure 1: Functional model of the target system in the form of a "black box"

AnyLogic was chosen to build the model based on the results of multicriteria analysis of simulation tools. This simulation system is superior in functionality to the alternative despite the rather high cost of an official license.

AnyLogic allows you to use several types of modeling at the same time. The elements of discrete-event (construction of a process diagram) and agent modeling (setting behavior of agents) are used in the developed model. [3] The table 1 shows the main elements of the model - agents and resources.

The following function blocks from the Process Modeling Library were used in the AnyLogic program to show the service process of customer: Source, Sink, Move To, Select Output, Service, Resource Pool [4] 
TABLE 1: Description of the main elements of the model

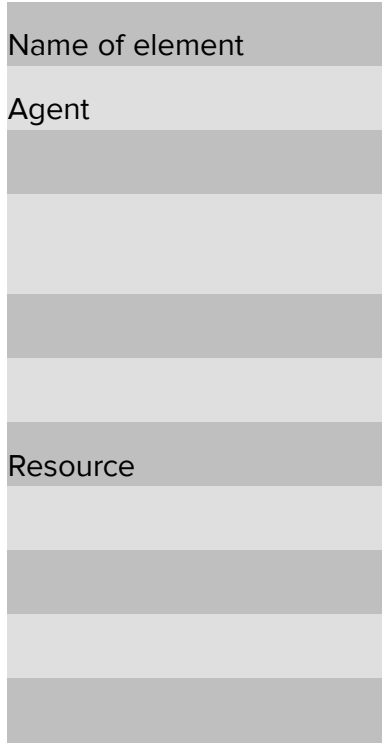

Attribute
Name
Path velocity
Input frequency
3D / 2D image availability
Behavior is set
Name
Path velocity
Quantity per unit time
3D / 2D image availability
Behavior is set

Value
Customer
$0,5 \mathrm{~m} / \mathrm{s}$
It is set by the schedule of intensity from
the database
3D, animation
Sequential Function Blocks
Cashier, Seller
$1 \mathrm{~m} / \mathrm{s}$
It is set by the schedule from the database
$3 \mathrm{D}$
Resource Pool

As the object of modeling, different types of outlets can be used. For example, stores may differ in the way they serve customers and the presence / absence of certain staffing units: with universal sellers; with sellers and cashiers. In the simplest case, when the store doesn't have a large staff of employees, universal sellers work on the trading floor. It is impractical to allocate the position of a cashier, since, if necessary, the seller can both advise the client and work at the cashier in such a situation.

In the case when the staff is represented by two categories of employees, the model will differ in the presence of two additional blocks: ResourcePool ("Cashiers") and Schedule ("cash_table").

The source data must be converted to the desired format before loading into the model. So the schedule file needed to be converted as follows (Figure 2).
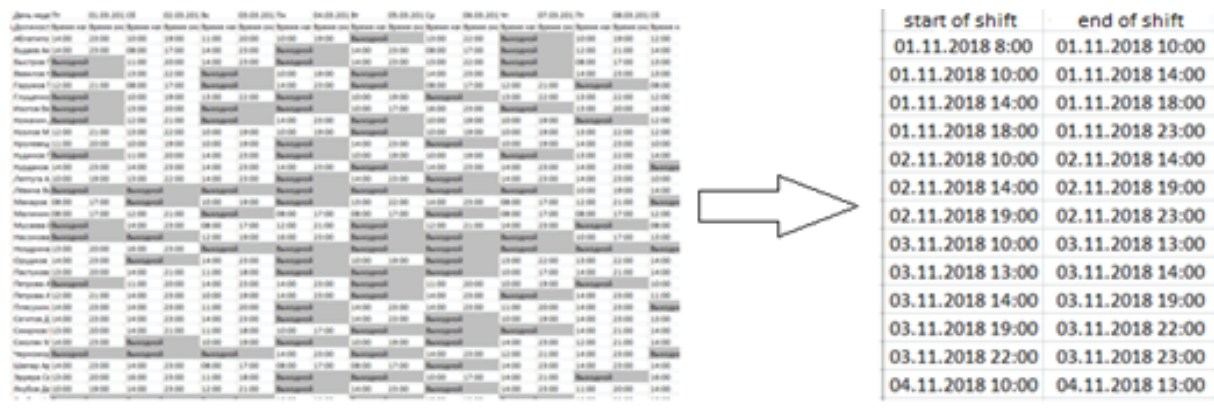

\begin{tabular}{c} 
number of sellers \\
1 \\
2 \\
\hline 3 \\
2 \\
1 \\
3 \\
\hline 2 \\
\hline 2 \\
\hline 3 \\
\hline 4 \\
\hline 2 \\
\hline 1 \\
\hline 2
\end{tabular}

Figure 2: Example of data conversion

After loading the data into the model and setting all the parameters necessary for the model to work, compilation is performed. The AnyLogic compiler checks the model 
for logical and syntax errors. If verification is successful, a simulation experiment is launched.

When the model is launched, customers arrive at the outlet according to the schedule of intensities specified earlier. There are 4 exits in the model: Exit with purchases, Exit after consultation, Exit from the cash, Exit without purchase - corresponding to possible outcomes of the service process. Variables store the number of customers leaving the outlet through each of exits.

Data sets are used to collect statistics. For clarity, particularly important indicators are displayed on graphs and charts. Thanks to the available dynamic indicators for each block, you can see the input and output values, as well as statistics during the simulation.

The figures below show the results: a block diagram with indicators (Figure 3).

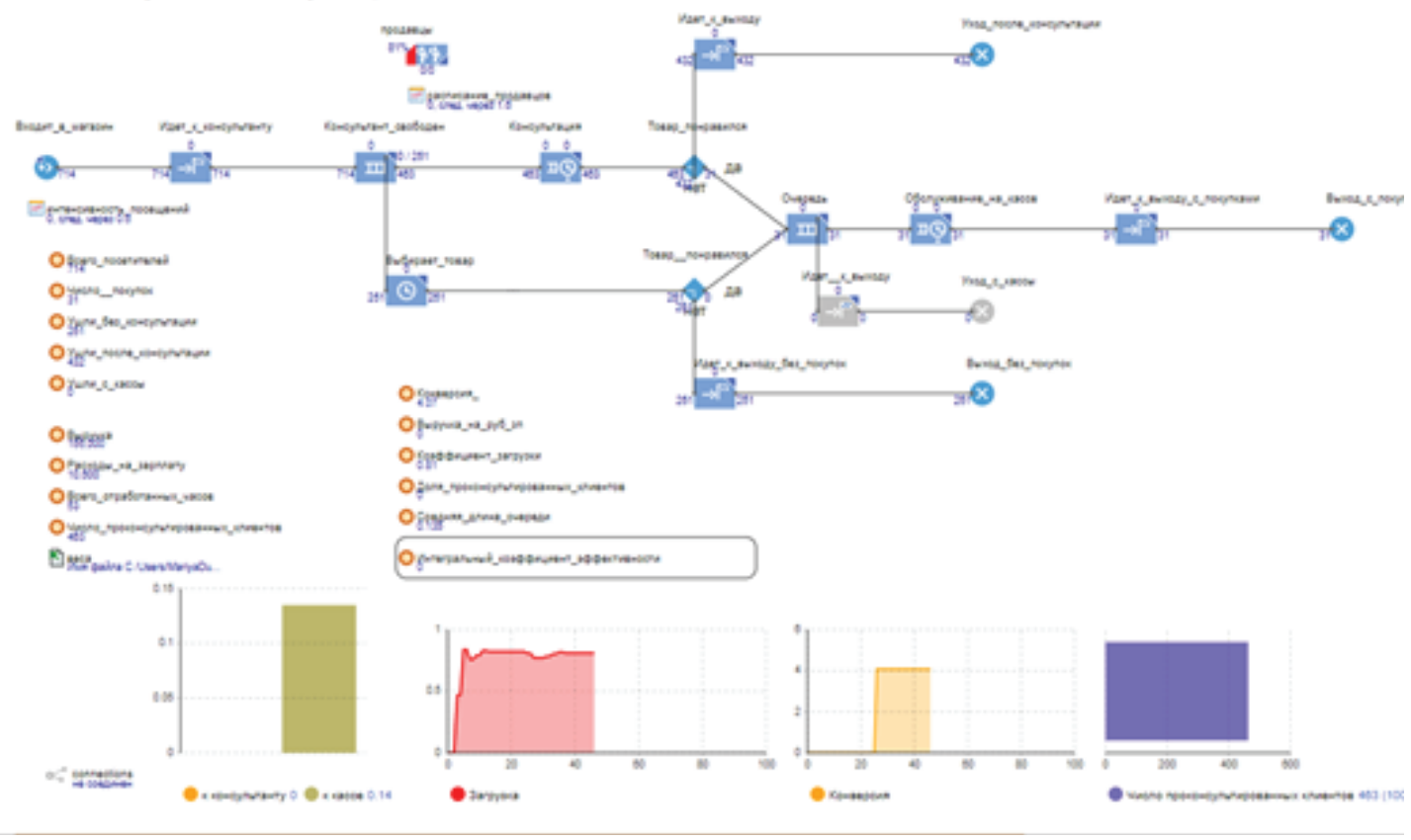

Figure 3: Scheme with indicators

\section{Conclusions}

After checking for compliance with the requirements and needs of stakeholders described separately, the resulting model can be used to validate WFM mathematical algorithms, as well as to present its advantages to the customer.

Also planned to add new factors to the model that determine the behavior of the client in order to bring it as close as possible to the object of modeling. 
And as the next step necessary to improve the data conversion process by its automation. One of the specialized programs for working with data can be used for this.

\section{References}

[1] K. K. Vasiliev, M. N. Serving. Mathematical modeling of communication systems: a training manual - Ulyanovsk: UISTU, 2008. - 170 p.

[2] Kossiakoff A. et al. Systems engineering principles and practice. - John Wiley \& Sons, 2011. - $83 \mathrm{p}$.

[3] Grigoriev, I. AnyLogic for three days: a practical guide on simulation modeling, 2017. $-273 p$.

[4] Blocks of the Process Modeling Library // Help - AnyLogic Simulation Software. [Electronic resource]. - Access mode: https://help.anylogic.ru/index.jsp?topic=\{\%\}2Fcom. anylogic.help\{\%\}2Fhtml\{\%\}2F_ProcessModeling\{\%\}2FPML_Blocks.html, free 